\title{
The Hypocretin/Orexin System Mediates the Extinction of Fear Memories
}

\author{
África Flores', Victòria Valls-Comamala',3, Giulia Costa², Rocío Saravia', Rafael Maldonado' and \\ Fernando Berrendero*, \\ 'Laboratory of Neuropharmacology, Department of Experimental and Health Sciences, Universitat Pompeu Fabra, PRBB, C/ Doctor Aiguader \\ 88, Barcelona, Spain; ²Department of Biomedical Sciences, Section of Neuropsychopharmacology, University of Cagliari, Cagliari, Italy
}

\begin{abstract}
Anxiety disorders are often associated with an inability to extinguish learned fear responses. The hypocretin/orexin system is involved in the regulation of emotional states and could also participate in the consolidation and extinction of aversive memories. Using hypocretin receptor-I and hypocretin receptor-2 antagonists, hypocretin-I and hypocretin-2 peptides, and hypocretin receptor-I knockout mice, we investigated the role of the hypocretin system in cue- and context-dependent fear conditioning and extinction. Hypocretins were crucial for the consolidation of fear conditioning, and this effect was mainly observed in memories with a high emotional component. Notably, after the acquisition of fear memory, hypocretin receptor-I blockade facilitated fear extinction, whereas hypocretin-I administration impaired this extinction process. The extinction-facilitating effects of the hypocretin receptor-I antagonist SB334867 were associated with increased expression of cFos in the basolateral amygdala and the infralimbic cortex. Intra-amygdala, but neither intra-infralimbic prefrontal cortex nor intra-dorsohippocampal infusion of SB334867 enhanced fear extinction. These results reveal a key role for hypocretins in the extinction of aversive memories and suggest that hypocretin receptor- I blockade could represent a novel therapeutic target for the treatment of diseases associated with inappropriate retention of fear, such as post-traumatic stress disorder and phobias.

Neuropsychopharmacology (2014) 39, 2732-274I; doi:I0.1038/npp.20 I4.I46; published online I6 July 2014
\end{abstract}

\section{INTRODUCTION}

The formation of aversive memories after stressful situations is an adaptive phenomenon necessary for individuals to survive. However, deregulation of acquired fear, revealed by enhanced conditioning or resistance to fear extinction, is associated with anxiety disorders, such as post-traumatic stress disorder (PTSD) and phobias (Holmes and Singewald, 2013). The knowledge of the neural substrate underlying conditioning and extinction of fear is particularly relevant for the identification of novel potential treatments for these pathologies (Tronson et al, 2012). Human and animals develop robust fear of environmental contexts or cues paired with aversive events. In rodent models, animals learn to associate an aversive unconditioned stimulus (US) such as a brief footshock with a neutral conditioned stimulus (CS), typically a chamber or a tone. In these models, extinction learning is usually slower than fear learning and usually requires multiple nonrein-

* Correspondence: Dr F Berrendero, Laboratory of Neuropharmacology, Department of Experimental and Health Sciences, University Pompeu Fabra, PRBB, C/ Doctor Aiguader 88, 08003 Barcelona, Spain, Tel: +34 93 3160890, Fax: +3493 3160901,

E-mail: fernando.berrendero@upf.edu

${ }^{3}$ Present address: Laboratory of Molecular Physiology and Channelopaties, Department of Experimental and Health Sciences, Universitat Pompeu Fabra, PRBB, C/ Doctor Aiguader 88, 08003 Barcelona, Spain. Received 25 February 20।4; revised I5 May 2014; accepted 9 June 2014; accepted article preview online 16 June 2014 forced expositions to the fear associated cues or context (Myers and Davis, 2007).

Hypocretin-1/orexin-A and hypocretin-2/orexin-B are lateral hypothalamic neuropeptides that project throughout the brain (de Lecea et al, 1998; Sakurai et al, 1998) and bind two different $\mathrm{G}$ protein-coupled receptors (Hcrtr-1 and Hcrtr-2) (de Lecea et al, 1998; Sakurai et al, 1998). Hypocretin-1 binds to both receptors with similar affinity, whereas hypocretin-2 binds to Hcrtr-2 with a 10 -fold greater affinity than Hcrtr-1. The hypocretin system modulates diverse physiological functions including sleep/arousal, energy homeostasis, and reward processing (Li et al, 2014). A role for hypocretins in emotional regulation has been recently proposed, consistent with the existence of hypocretin neuronal projections to several limbic areas (Johnson et al, 2012). In agreement, clinical research showed a reduced amygdala activity during aversive conditioning in human narcolepsy (Ponz et al, 2010), a condition associated with a loss of hypocretin neurons (Peyron et al, 2000). Moreover, human subjects with panic anxiety have elevated hypocretin levels in the cerebrospinal fluid (Johnson et al, 2010), and activation of hypocretin-synthesizing neurons is necessary for developing a panic-prone state in rodent models (Johnson et al, 2010). In agreement, the dual hypocretin receptor antagonist almorexant reduced fear-conditioned startle reactions in the rat (Steiner et al, 2012).

The aim of this study was to investigate the involvement of the hypocretin system in the consolidation and extinction of aversive memories. We also evaluated the brain regions 
mediating hypocretin effects in fear extinction by using cFos expression. Finally, we infused the Hcrtr-1 antagonist SB334867 into the amygdala, infralimbic prefrontal cortex (IL) and the dorsal hippocampus to confirm the involvement of specific brain areas in this extinction process.

\section{MATERIALS AND METHODS}

\section{Animals}

Experiments were performed in male C57BL/6J mice (Charles River, France), 129S1/SvImJ mice (Jackson Laboratories, USA) and Hcrtr-1 knockout (KO) mice and their wild-type (WT) littermates (Jackson Laboratories) (8-12 weeks old). KO mice were backcrossed for at least seven generations to $\mathrm{C} 57 \mathrm{BL} / 6 \mathrm{~J}$ mice, and genotyped as described previously (Flores et al, 2014). Mice were housed four per cage in a temperature- $\left(21^{\circ} \mathrm{C} \pm 1{ }^{\circ} \mathrm{C}\right)$ and humidity-controlled $(55 \pm 10 \%)$ room with a $12 \mathrm{~h}$ light/dark cycle (light between 0800 and 2000 hours). Food and water were available ad libitum. All animal procedures were conducted in accordance with the guidelines of the European Communities Directive 86/609/EEC regulating animal research and approved by the local ethical committee (CEAA-PRBB) and the statement of compliance with standards for use of laboratory animals by foreign institutions (\#A5388-01 approved by the National Institutes of Health). All experiments were performed under blind conditions.

\section{Drugs}

The Hcrtr-1 antagonist SB334867 (3, 5 and $10 \mathrm{mg} / \mathrm{kg}$ ) (Tocris) was dissolved in 1\% (wt/vol) 2-hydroxypropyl- $\beta$ cyclodextrin (Sigma) and 10\% dimethyl sulfoxide (vol/vol) in distilled water. The Hcrtr-2 antagonist TCSOX229 (5, 10 and $15 \mathrm{mg} / \mathrm{kg}$ ) (Tocris) was dissolved in physiological saline. Both hypocretin antagonists were administered by intraperitoneal (i.p.) route in a volume of $5 \mathrm{ml} / \mathrm{kg}$ body weight. The SB334867 and TCSOX229 doses were based on previous studies and did not modify spontaneous locomotor activity (Plaza-Zabala et al, 2010, 2012). Hypocretin-1 and hypocretin-2 (Sigma) were dissolved in physiological saline and a volume of $1 \mu \mathrm{l}$ was administered by intracerebroventricular (i.c.v.) route at 0.75 and $2.5 \mathrm{nmol} / \mu \mathrm{l}$, respectively. These doses were based on previous studies (Plaza-Zabala et al, 2012; Boutrel et al, 2005). For intracerebral microinjections, SB334867 (10 nmol/side) was dissolved in a 25:75 sterile saline solution:dimethyl sulfoxide vehicle. This dose was based on preceding reports (Hollander et al, 2008; Narita et al, 2006). Ketamine hydrochloride (100 mg/kg) (Imalgène 1000, Merial) and xylazine hydrocloride $(20 \mathrm{mg} / \mathrm{kg}$ ) (Ronpum, Sigma) were mixed and dissolved in ethanol (5\%) and distilled water (95\%). This anesthetic mixture was administered i.p. in a volume of $10 \mathrm{ml} / \mathrm{kg}$ body weight.

\section{Behavioral Experiments}

Contextual fear conditioning and extinction. Mice were fear-conditioned in a shuttle chamber (LE918, Panlab, Barcelona) surrounded by a larger sound-attenuating cabinet, which had a ventilation fan to provide background noise. A speaker attached to the wall of the chamber delivered the tone stimuli. The shuttle chamber floor was constructed of parallel stainless-steel bars and connected to a scrambled shock generator (LE10026, Panlab). Before each trial, the chamber was cleaned with $70 \%$ ethanol and then with water to remove olfactory cues. On the training day, mice were placed in the conditioning chamber for $150 \mathrm{~s}$ before the exposure to an US $(0.6 \mathrm{~mA}$ footshock for $2 \mathrm{~s})$ and then remained in the chamber for $30 \mathrm{~s}$. Different experimental designs were used to evaluate the consolidation and the extinction of contextual fear memories. To study the consolidation process, pharmacological treatments were administered immediately after the training session. Testing was performed $24 \mathrm{~h}$ after training by returning mice to the conditioning chamber and measuring the freezing behavior for $3 \mathrm{~min}$. Freezing, a rodent's natural response to fear (LeDoux, 1993), was defined as the absence of all movement except for that necessary for breathing and was scored manually. To evaluate the extinction of contextual fear memories, no pharmacological treatments were administered after the training session to preserve fear memory formation. Extinction trials (E1-E5) were performed at 24, $48,72,96$, and $120 \mathrm{~h}$ after the training by scoring freezing behavior for $3 \mathrm{~min}$ in the conditioning chamber. Pharmacological treatments were administered immediately after each extinction session.

Cued fear conditioning and extinction. A different group of mice underwent a cued fear conditioning (FC). On the training day, each mouse was placed in the conditioning chamber previously described for $120 \mathrm{~s}$ before the onset of three cue tones $(3 \mathrm{kHz}, 90 \mathrm{~dB}, 8 \mathrm{~s}$ long, $2 \mathrm{~s}$ between tones) each one co-terminating with the delivery of one footshock $(0.6 \mathrm{~mA}, 2 \mathrm{~s})$, and then remained in the chamber for $30 \mathrm{~s}$. This cue tone exposure did not induce freezing behavior by itself (Supplementary Figure S1). To study the consolidation process, pharmacological treatments were administered immediately after the training trial and testing was performed $24 \mathrm{~h}$ after training by placing mice in a different context (a transparent Plexiglas cylinder) for $6 \mathrm{~min}$. The first 3-min mice were habituated to the new context, and during the following $3 \mathrm{~min}$ animals were re-exposed to the CS (18 cue tones, $8 \mathrm{~s}$ long, $2 \mathrm{~s}$ between tones). Freezing behavior was scored during the first $2 \mathrm{~min}$ of cue re-exposure. To evaluate the extinction of cued fear memories, mice were placed in the transparent cylinder at 24,48 , and $72 \mathrm{~h}$ after the footshock (extinction sessions E1-E4), and freezing behavior was evaluated as previously described. Pharmacological treatments were administered immediately after each extinction trial.

Novel object-recognition test. See Supplementary Materials and Methods.

Locomotor activity, Hot-plate test, and Light-dark box. Locomotion, nociception, and anxiety-like behavior were evaluated in Hcrtr-1 KO mice. See Supplementary Materials and Methods.

Elevated plus-maze and Open-field test. Locomotion and anxiety-like behavior were evaluated in mice $24 \mathrm{~h}$ after i.p. injections of SB334867 (3 and $5 \mathrm{mg} / \mathrm{kg}$ ), TCSOX229 (10 and 
$15 \mathrm{mg} / \mathrm{kg})$, hypocretin-1 $(0.75 \mathrm{nmol} / \mu$, i.c.v. $)$, or respective vehicles. The following experimental sequence was performed: locomotor activity boxes $24 \mathrm{~h}$ after injection, elevated plus-maze (15 min later), and open-field test (30 min later). See Supplementary Materials and Methods.

\section{Stereotaxic Surgery and Infusion Procedure}

Mice were implanted with unilateral cannulae in the lateral ventricle for i.c.v. administration of hypocretin-1 and hypocretin-2 to evaluate the role of these peptides in fear extinction. In a different group of mice, bilateral cannulae were implanted in the amygdala, the IL, or the hippocampus, and SB334867 microinjections were performed in these specific brain regions during contextual fear extinction. See Supplementary Materials and Methods.

\section{Immunofluorescence Studies}

See Supplementary Materials and Methods.

\section{Statistical Analysis}

One-way analysis of variance (ANOVA) was used to analyze consolidation of fear memory experiments and the activation of hypocretin neurons during fear extinction. Two-way ANOVA of repeated measures was used to analyze fear extinction behavioral data and cFos expression during fear extinction under SB334867 treatment. Subsequent post hoc comparison (Fisher's LSD) was employed when required. All data were analyzed using Statistica (StatSoft) software. The level of significance was $p<0.05$ in all experiments.

\section{RESULTS}

\section{Hcrtr-1 and Hcrtr-2 Contribute Differently in the Consolidation of fear Memories}

To evaluate the possible role of the hypocretinergic system in the consolidation of fear memories, we pharmacologically blocked Hcrtr-1 and Hcrtr-2 activity in contextual and cued FC paradigms. Mice were treated with selective Hcrtr-1 and Hcrtr-2 antagonists immediately after the FC session, and $24 \mathrm{~h}$ later animals were tested for conditioned freezing behavior, a general reaction to perceived threat (Fanselow, 1980) (Figure 1a). Systemic administration of the selective Hcrtr-1 antagonist SB334867 (3, 5, and $10 \mathrm{mg} / \mathrm{kg}$, i.p.) significantly decreased the evoked freezing behavior when compared with the vehicle-treated group in both contextual $\left(\mathrm{F}_{3,32}=21.19, p<0.001\right)$ and cued $\left(\mathrm{F}_{3,22}=10.04, p<0.001\right)$ fear tests, at all doses used $(p<0.01)$ as revealed by post hoc comparison (Figure $1 \mathrm{~b}$ and $c$ ). Hcrtr-1 KO mice also displayed significant reduced levels of freezing during contextual $(p<0.05)$ and cued $(p<0.01)$ fear tests, confirming a role for Hcrtr-1 in fear memory formation (Figure $1 \mathrm{~d}$ and e). WT and Hcrtr-1 KO mice did not show differences in pain perception, anxiety-related behavior, and locomotor activity (Supplementary Figure S2). In contrast, systemic injection of the selective Hcrtr-2 antagonist $\operatorname{TCSOX} 229(5,10$ and $15 \mathrm{mg} / \mathrm{kg}$, i.p. $)$ shortened the freezing time during contextual fear test $\left(\mathrm{F}_{3,45}=4.62\right.$, $p<0.001)$ at the doses of 10 and $15 \mathrm{mg} / \mathrm{kg} \quad(p<0.01)$ (Figure 1f), but did not interfere with cued fear consolidation
$\left(\mathrm{F}_{3,19}=1.07\right.$, NS) (Figure 1g). These results suggest that Hcrtr-2 is selectively involved in contextual fear memory consolidation, in agreement with recent findings supporting a different role for Hcrtr-1 and Hcrtr-2 in the formation of contextual and cued fear memories (Soya et al, 2013). Interestingly, SB334567 and TCSOX229 administration $4 \mathrm{~h}$ after the FC trial (Figure 1h) did not affect the context- or cued-conditioned freezing response (Figure 1i-k), indicating that Hcrtr-1 and Hcrtr-2 signaling is recruited immediately after contextual and cued FC during the consolidation of fear memories.

To further clarify the specific involvement of Hcrtr-1 and Hcrtr-2 in the consolidation of emotional memories, we used the novel object-recognition paradigm (Figure 11), which reflects declarative memory with a low emotional component. In contrast to $\mathrm{FC}$, systemic administration of SB334867 immediately after training did not modify memory consolidation $\left(\mathrm{F}_{3,40}=1.94\right.$, NS) (Figure $\left.1 \mathrm{~m}\right)$, although a tendency to decrease the discrimination index was observed at the higher dose $(10 \mathrm{mg} / \mathrm{kg})$. In agreement, Hcrtr-1 KO mice exhibited normal memory performance in the novel object-recognition test (Figure 1n). TCSOX229 did not modify the discrimination index in this behavioral paradigm (Figure 10). Total object exploration time was not altered in any of the different experiments performed (Supplementary Figure S3). These results suggest that hypocretins could be mainly involved in the consolidation of highly emotional memories.

\section{Hcrtr-1 Blockade Facilitates the Extinction of Fear Memories}

To assess the involvement of hypocretins in contextual and cued fear extinction, mice underwent a single FC-training trial and did not receive treatment to preserve fear memory formation. Freezing response was scored $24 \mathrm{~h}$ later in a first extinction session (E1). Animals were assigned to a treatment group (balanced for the freezing levels) and treated with hypocretin receptor agonists or antagonists immediately after the first extinction session and daily after each ensuing extinction trial (Figure 2a). Daily administration of SB334867 (3 and $5 \mathrm{mg} / \mathrm{kg}$, i.p.) significantly accelerated the context extinction process (treatment effect: $\mathrm{F}_{2,37}=4.61, \quad p<0.05$; interaction treatment $\times$ day: $\mathrm{F}_{8,148}$ $=3.03, p<0.01$ ) (Figure 2b). Post hoc analyses showed a significant effect of SB334867 at the doses of $3 \mathrm{mg} / \mathrm{kg}$ $(p<0.05$ at E2, $p<0.01$ at E3-E5) and $5 \mathrm{mg} / \mathrm{kg}(p<0.05$ at E2-E5). Consistently, the i.c.v. administration of hypocretin-1 $(0.75 \mathrm{nmol} / \mu \mathrm{l})$ after each extinction session impaired the contextual extinction process (treatment effect: $\mathrm{F}_{1,12}=24.12, p<0.001$; interaction treatment $\times$ day: $\mathrm{F}_{4,48}=27.73, p<0.001$ ) (Figure 2d). Post hoc comparisons revealed significant differences between vehicle- and hypocretin-1-treated groups from E3 to E5 $(p<0.05$ at E3, $p<0.01$ at E4-E5). In contrast, the administration of TCSOX229 (Figure 2c) (10 and $15 \mathrm{mg} / \mathrm{kg}$, i.p.) and hypocretin-2 $(2.5 \mathrm{nmol} / \mu \mathrm{l})$ (Figure $2 \mathrm{e})$ after each extinction trial did not modify contextual memory extinction (interaction treatment $\times$ day: $\mathrm{F}_{8,80}=0.51$, NS and $\mathrm{F}_{4,36}=0.32$, NS, respectively). Similar results were obtained in the cued fear extinction paradigm. Thus, SB334867 (3 and $5 \mathrm{mg} / \mathrm{kg}$, i.p.) administration after each extinction trial enhanced cued 

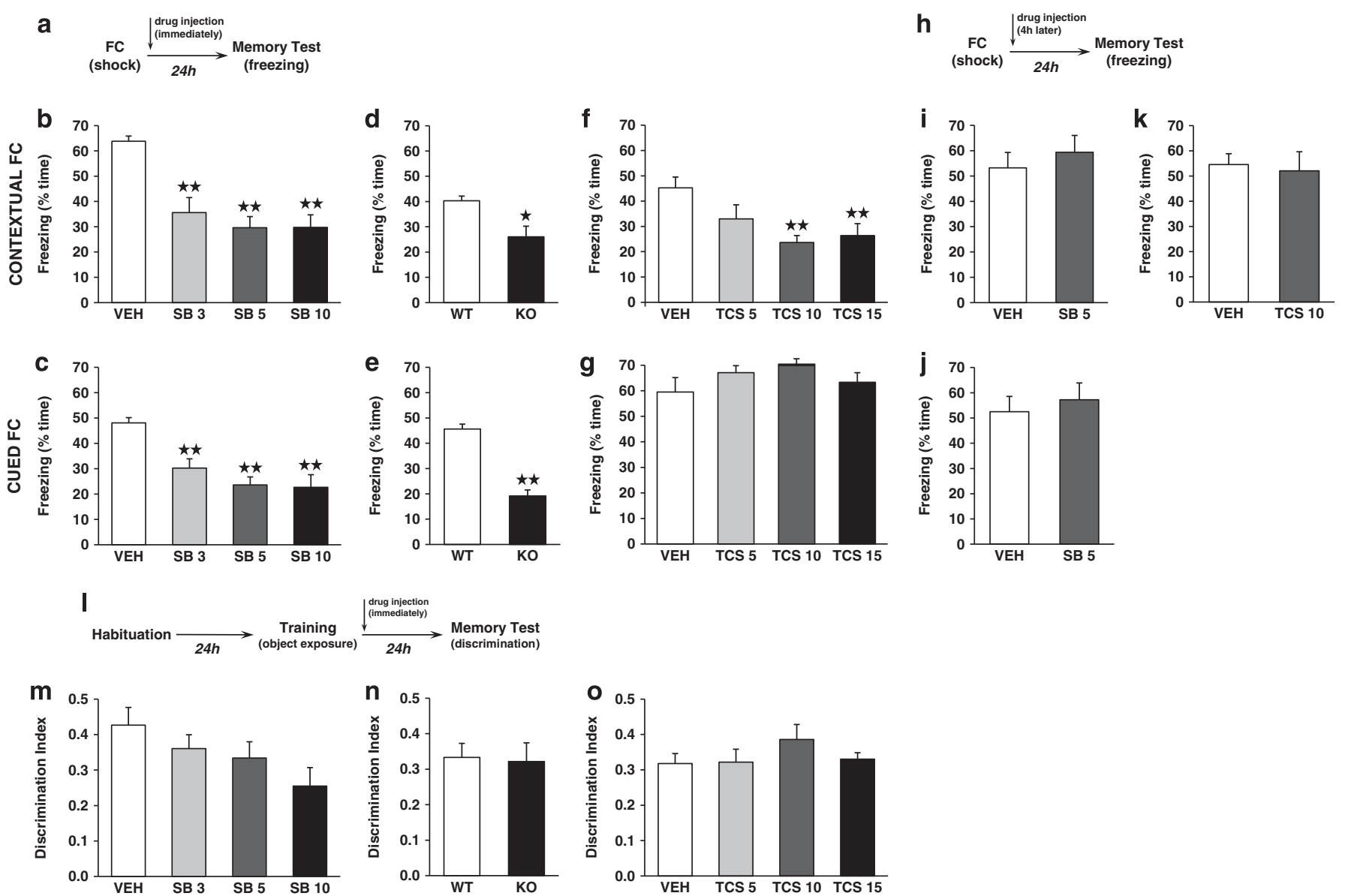

Figure I Hcrtr-I and Hcrtr-2 modulate differently context- and cue-induced fear memory consolidation. (a) Schematic representation of the experimental design for $(\mathrm{b}-\mathrm{g})$ behavioral tests. $(\mathrm{b}-\mathrm{g})$ Freezing levels scored during the $(\mathrm{b}, \mathrm{d}$ and $\mathrm{f}$ ) contextual and (c,e and $\mathrm{g})$ cued memory test in mice treated with (b and c) SB334867 (3, 5 and 10 mg $/ \mathrm{kg}$, i.p.) or vehicle ( $n=6-14$ mice per group) and with (f, g) TCSOX229 (5, 10 and I 5 mg $/ \mathrm{kg}$, i.p.) or vehicle ( $n=6-13$ mice per group) immediately after the FC trial, as well as in (d and e) Hcrtr-I KO and WT mice ( $n=7-9$ mice per group). (h) Schematic representation of the experimental design for $(i-j)$ behavioral tests. (i-k) Freezing levels scored during the $(i$ and $k)$ contextual or $(j)$ cued memory test in mice ( $n=6$ mice per group) injected $4 \mathrm{~h}$ after the FC session with ( $\mathrm{i}$ and $\mathrm{j})$ SB334867 (3, 5 and $10 \mathrm{mg} / \mathrm{kg}$, i.p.) or vehicle and with ( $\mathrm{k}$ ) TCSOX229 (5, I0 and $15 \mathrm{mg} / \mathrm{kg}$, i.p.) or vehicle. (I) Schematic representation of the experimental design for $(\mathrm{m}-\mathrm{O})$ behavioral tests. ( $\mathrm{m}-\mathrm{O})$ Discrimination index obtained in the novel object-recognition paradigm by mice treated with $(\mathrm{m})$ SB334867 (3, 5 and $10 \mathrm{mg} / \mathrm{kg}$, i.p.) or vehicle $(n=8-14$ mice per group) and with (o) $\operatorname{TCSOX} 229(5,10$ and $15 \mathrm{mg} / \mathrm{kg}$, i.p.) or vehicle $(n=8-14$ mice per group) immediately after the training, as well as in ( $\mathrm{n}) \mathrm{Hcrtr}-1 \mathrm{KO}$ and WT mice ( $n=8$ mice per group). Data are expressed as mean \pm SEM. $* p<0.05$, ** $p<0.01$ compared with the control group. FC, fear conditioning; VEH, vehicle; SB, SB334867; TCS, TCSOX229; WT, wild-type mice; KO, Hcrtr-I knockout mice.

fear extinction (treatment effect: $\mathrm{F}_{2,20}=4.13, p<0.05$; interaction treatment $\times$ day: $F_{6,60}=3.84, p<0.01$ ) (Figure $2 \mathrm{f}$ ). Subsequent post hoc analysis revealed significant differences between vehicle- and SB334867-treated animals only at E2 ( 3 and $5 \mathrm{mg} / \mathrm{kg}: p<0.01$ ), probably due to the fast extinction exhibited by the vehicle group in these experimental conditions. In agreement, daily administration of hypocretin-1 $(0.75 \mathrm{nmol} / \mu$ l, i.c.v. $)$ delayed extinction of freezing behavior (treatment effect: $\mathrm{F}_{1,13}=22.01, p<0.001$; interaction treatment $\times$ day: $\mathrm{F}_{3,39}=5.99, p<0.01$ ) (Figure $2 \mathrm{~h}$ ), and significant differences were revealed from E2 to E4 $(p<0.05$ at E2; $p<0.01$ at E3-E4). On the contrary, administration of either TCSOX229 (10 and $15 \mathrm{mg} / \mathrm{kg}$, i.p.) or hypocretin-2 $(2.5 \mathrm{nmol} / \mu \mathrm{l}$, i.c.v. $)$ did not modify cued fear extinction (interaction treatment $\times$ day: $\mathrm{F}_{6,63}=0.50$, NS and $\mathrm{F}_{3,36}=$ 0.78 , NS, respectively) (Figure $2 \mathrm{~g}$ and i). Locomotor activity and anxiety-like responses were not modified in mice $24 \mathrm{~h}$ after the acute administration of SB334867 (3 and $5 \mathrm{mg} / \mathrm{kg}$, i.p.), TCSOX229 (5 and $10 \mathrm{mg} / \mathrm{kg}$, i.p.), and hypocretin-1
$(0.75 \mathrm{nmol} / \mu \mathrm{l}$, i.c.v.) (Supplementary Figure S4), suggesting that the changes observed in freezing behavior by hypocretin compounds were not due to unspecific effects on locomotion and anxiety.

To further study the role of Hcrtr-1 in fear extinction, we tested the extinction-facilitating effects of SB334867 in the $129 \mathrm{~S} 1 / \mathrm{SvImJ}$ inbred mouse strain, a well-established mouse model of impaired extinction (Hefner et al, 2008). Administration of SB334867 $(5 \mathrm{mg} / \mathrm{kg}$, i.p.) after each extinction trial rescued contextual (treatment effect: $\mathrm{F}_{1,11}=$ 46.98, $p<0.001$; interaction treatment $\times$ day: $F_{4,44}=8.04$, $p<0.01$ ) (Figure 2j), as well as cued fear extinction in these mice (treatment effect: $F_{1,22}=9.97, p<0.01$; interaction treatment $\times$ day: $\mathrm{F}_{3,66}=7.09, p<0.001$ ) (Figure $2 \mathrm{k}$ ). Post hoc comparisons revealed significant differences between vehicle and SB334867 groups from E2 to E5 in contextual extinction $(p<0.01)$ and from E3 to E4 in cued extinction $(p<0.05)$, confirming the extinction-enhancing effects of Hcrtr-1 blockade. 


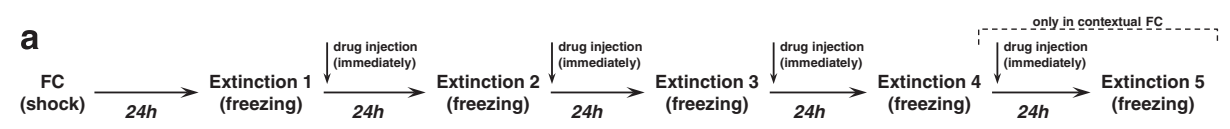

\section{CONTEXTUAL FC}
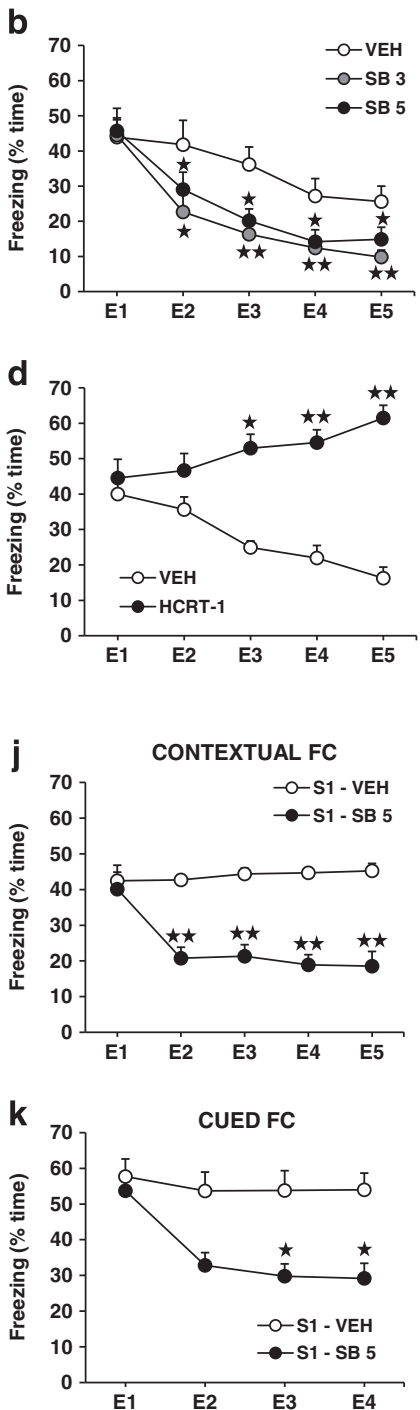
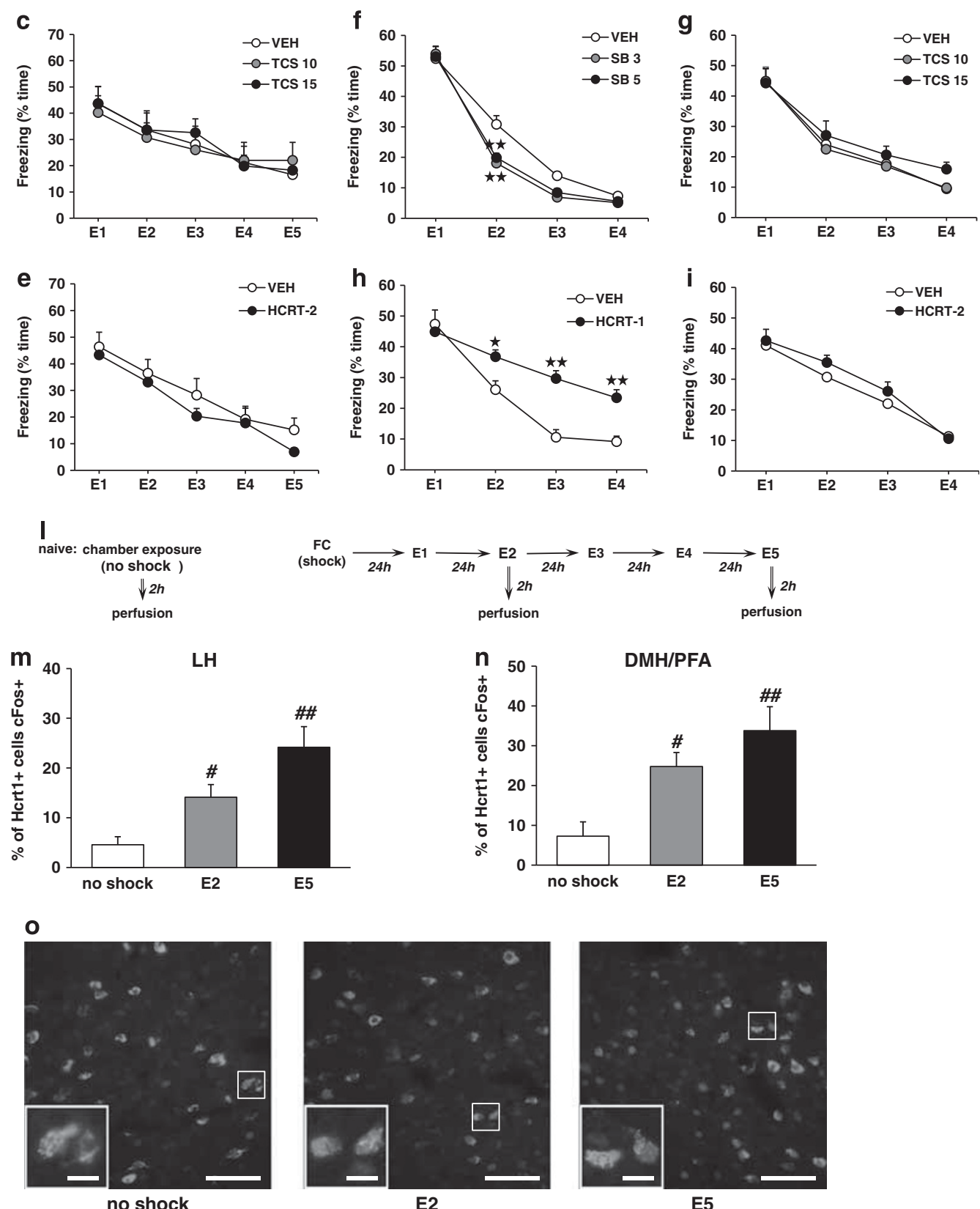

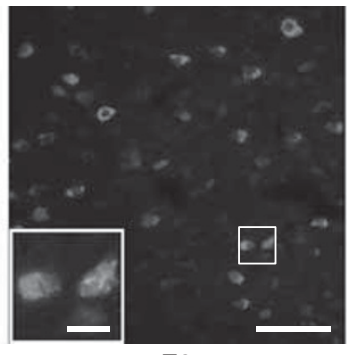

E2

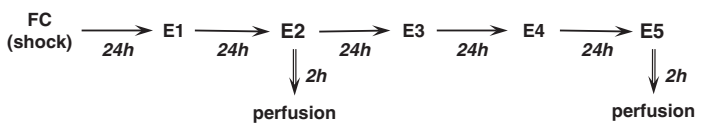

DMH/PFA
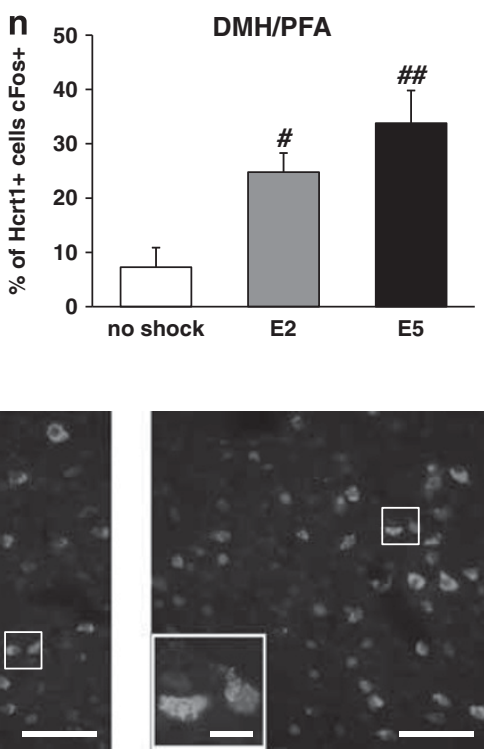

E5

Figure 2 Hypocretin transmission is involved in the extinction of fear memories through Hcrtr-I signaling. (a) Schematic representation of the experimental design for $(b-k)$ tests. (b-i) Time course of the freezing levels scored during $(b-e)$ contextual and (f-i) cued extinction trials in mice treated immediately after each extinction session with (b and f) SB334867 (3 and $5 \mathrm{mg} / \mathrm{kg}$, i.p.) or vehicle ( $n=7-14$ mice per group), (c and g) TCSOX229 (I0 and $15 \mathrm{mg} / \mathrm{kg}$, i.p.) or vehicle ( $n=7-8$ mice per group), ( $\mathrm{d}$ and $\mathrm{h})$ hypocretin- I $(0.75 \mathrm{nmol} / \mu \mathrm{l}$, i.c.v.) or vehicle ( $n=6-8 \mathrm{mice}$ per group), and (e and i) hypocretin$2(2.5 \mathrm{nmol} / \mu$ l, i.c.v.) or vehicle $(n=6-7$ per group). ( $j$ and $k)$ Time course of the freezing levels scored during (j) contextual and ( $k$ ) cued extinction trials in I29SI/SvImJ mice treated with SB334867 (5 mg/kg, i.p.) or vehicle $(n=7-13$ mice per group) immediately after each extinction session. (I) Schematic representation of the experimental design for $(m-0)$ biochemical experiments. ( $m$ and $n$ ) Percentage of hypocretin cells expressing cFos in the $(m) \mathrm{LH}$ and the (n) DMH/PFA of mice exposed to the context without receiving any footshock, and mice subjected to FC and subsequent extinction until E2 and E5 ( $n=5-7$ mice per group). (o) Representative images of the DMH/PFA obtained via fluorescence microscopy after direct double labeling combining rabbit polyclonal antiserum to cFos with mouse monoclonal antibody to hypocretin- I. The scale bars represent $40 \mu \mathrm{m}$ in general images and $10 \mu \mathrm{m}$ in magnified images. Data are expressed as mean \pm SEM. ${ }^{*} p<0.05$, *** $p<0.01$ compared with the vehicle group; ${ }^{*} p<0.05$, \#\# $p<0.01$ compared with the no-shock group. FC, fear conditioning; EI-E5, extinction trials I-5; VEH, vehicle; SB, SB334867; TCS, TCSOX229; HCRT-I, hypocretin- I; HCRT-2, hypocretin-2; SI, I29SI/SvImJ mice; LH, lateral hypothalamus; DMH/PFA, dorsomedial hypothalamus and perifornical area. A full color version of this figure is available at the Neuropsychopharmacology journal online. 
We next studied the possible activation of hypocretin neurons in the lateral hypothalamus (LH) and the dorsomedial hypothalamus and perifornical area (DMH/ PFA) during the contextual fear extinction process by using double-label immunofluorescence of cFos with hypocretin-1. C57BL/6J mice were exposed to fear extinction training, and brains were removed and perfused at different time points of the extinction process: naive mice (animals exposed to the chamber, but not to shock), $2 \mathrm{~h}$ after the extinction session 2 (E2) and $2 \mathrm{~h}$ after the last extinction trial (E5) (Figure 2l). The percentage of hypocretin neurons expressing cFos was increased during the extinction process in the LH $\left(\mathrm{F}_{2,15}=9.38, p<0.01\right)$ (Figure $2 \mathrm{~m})$ and the DMH/PFA $\left(\mathrm{F}_{2,15}=7.90, p<0.01\right)$ (Figure $2 \mathrm{n}$ and o). Post hoc analysis showed significant differences in comparison with basal conditions at E2 $(p<0.05)$ and E5 $(p<0.01)$ time points, suggesting that hypocretin neurons are recruited during fear extinction to counteract this process.

\section{The Extinction-Facilitating Effects of Hcrtr-1 Blockade are Associated with an Increased Activity of the Infralimbic Prefrontal Cortex and the Basolateral Amygdala}

To identify the brain areas responsible for the extinctionenhancing effect of Hcrtr-1 blockade, we analyzed the possible activation of the main brain regions involved in the extinction circuit of fear memories by using cFos immunofluorescence. Thus, cFos expression in prelimbic prefrontal cortex (PL), IL, dorsal hippocampus, and basolateral amygdala (BLA) was evaluated at different time points of the contextual extinction process (no shock, E2, and E5) in mice treated with vehicle or SB334867 $(5 \mathrm{mg} / \mathrm{kg}$, i.p.) immediately after each extinction trial (Figure 3a). PL showed a significant activation during contextual extinction at both E2 and E5 time points, which was not modified by SB334867 treatment (day effect: $F_{2,36}=38.63, p<0.001$; interaction day $\times$ treatment: $F_{2,36}=2.18$, NS) (Figure $3 b$ ). Similarly, dorsal hippocampus exhibited a significant increase in cFos expression during E2 and E5, which remained unaffected by SB334867 treatment (day effect: $F_{2,36}=5.09$, $p<0.01$; interaction day $\times$ treatment: $F_{2,36}=0.04, \quad$ NS) (Figure 3d). An increase in cFos expression was also observed in IL at E2 and E5 compared with the naive group (day effect: $\mathrm{F}_{2,36}=22.65, p<0.001$; interaction day $\times$ treatment: $\left.\mathrm{F}_{2,36}=3.83, p<0.05\right)$. Notably, SB334867 treatment induced a higher activation of this brain region at E2 $(p<0.01)$ in comparison with vehicle-treated animals (Figure $3 \mathrm{c}$ and $\mathrm{f}$ ). A similar effect was also observed in the BLA. This nucleus was significantly activated during extinction compared with control mice (day effect: $\mathrm{F}_{2,36}=19.13, p<0.001$; interaction day $\times$ treatment: $F_{2,36}=4.01, p<0.05$ ) (Figure $3 \mathrm{e}$ and $\mathrm{g}$ ), and SB334867 treatment enhanced cFos expression after the E2 extinction trial $(p<0.01)$. SB334867 did not modify cFos expression by itself in control no-shock animals (Figure $3 \mathrm{~b}-\mathrm{e})$. The blockade of Hcrtr-1 could have a more prominent effect at the beginning of the extinction process in agreement with the increase of cFos in IL and BLA on E2. Thus, a single injection of SB334867 (5 mg/kg, i.p., after the first extinction session) (Supplementary Figure S5) accelerated fear extinction on E2 and E3, although no significant differences were observed following E4 and E5. As a whole, these results show that the extinction-facilitating effects of Hcrtr-1 blockade are associated with an increased activity of the IL and the BLA, which are key regions involved in the extinction of aversive memories.

\section{Intra-Amygdala Infusion of SB334867 Facilitates Fear Extinction}

We next evaluated the possible direct participation of Hcrtr-1 in specific brain regions mediating the extinction of aversive memories. For this purpose, mice were bilaterally implanted with cannulae into the BLA, the IL, or the dorsal hippocampus and received intra-structure SB334867 microinjections ( $10 \mathrm{nmol} / 0.5 \mu \mathrm{l} / \mathrm{side})$ after each contextual extinction session. Blockade of Hcrtr-1 into the amygdala significantly accelerated context-induced fear extinction (treatment effect: $F_{1,28}=5.94, p<0.05$; interaction day $\times$ treatment: $\mathrm{F}_{4,112}=2.46, p<0.05$ ) (Figure $4 \mathrm{a}$ ). In contrast, SB334867 injections into the IL and the dorsal hippocampus had no effect in extinction of aversive memories (interaction day $\times$ treatment: $\mathrm{F}_{4,88}=0.94$, NS and $\mathrm{F}_{4,80}=0.89$, NS, respectively) (Figure $4 \mathrm{~d}$ and g). Figure $4 \mathrm{~b}$, e and h shows the location of the injection sites, and Figure 4c, $\mathrm{f}$ and i shows representative images of bilateral cannulae positions. These results suggest that the acceleration of fear extinction induced by SB334867 is partially mediated by Hcrtr-1 into the amygdala, whereas is independent of the activation of Hcrtr-1 in both the IL and the dorsal hippocampus.

\section{DISCUSSION}

Our data show that hypocretins have a crucial role in the consolidation of fear memories, whereas these neuropeptides are not involved in memories with a low emotional component. Notably, after the acquisition of fear, the Hcrtr-1 antagonist SB334867 facilitates extinction of aversive memories and increases the activity of relevant brain areas related to the extinction process, such as the BLA and the IL. Direct infusion of SB334867 into the amygdala also enhances fear extinction.

Pharmacological blockade with low doses of SB334867 immediately after the footshock, or genetic deletion of Hcrtr-1, impaired freezing responses in both cued and contextual FC paradigms. Consistent with previous reports, Hcrtr-1 KO mice showed similar pain perception, locomotor activity, and anxiety-like behavior than WT animals (Soya et al, 2013; Scott et al, 2011), suggesting that the differences between the two genotypes in fear memory formation are not due to alterations in sensory-motor abilities of the KO mice. In contrast, the administration of the Hcrtr-2 antagonist TCSOX229 only impaired contextual FC. These data show a different role for Hcrtr-1 and Hcrtr-2 in the consolidation of cued and contextual fear memories, in agreement with recent data using KO mice (Soya et al, 2013). The different location of these receptors could explain this divergent response. Thus, Hcrtr-2 is highly expressed in the hippocampus (Johnson et al, 2012), which has a critical role in the consolidation of contextual fear memories (Maren et al, 2013), but its expression in amygdala is minimal, which is involved in both processes (Maren et al, 2013; Flavell and Lee, 2012). Hcrtr-1 is located 


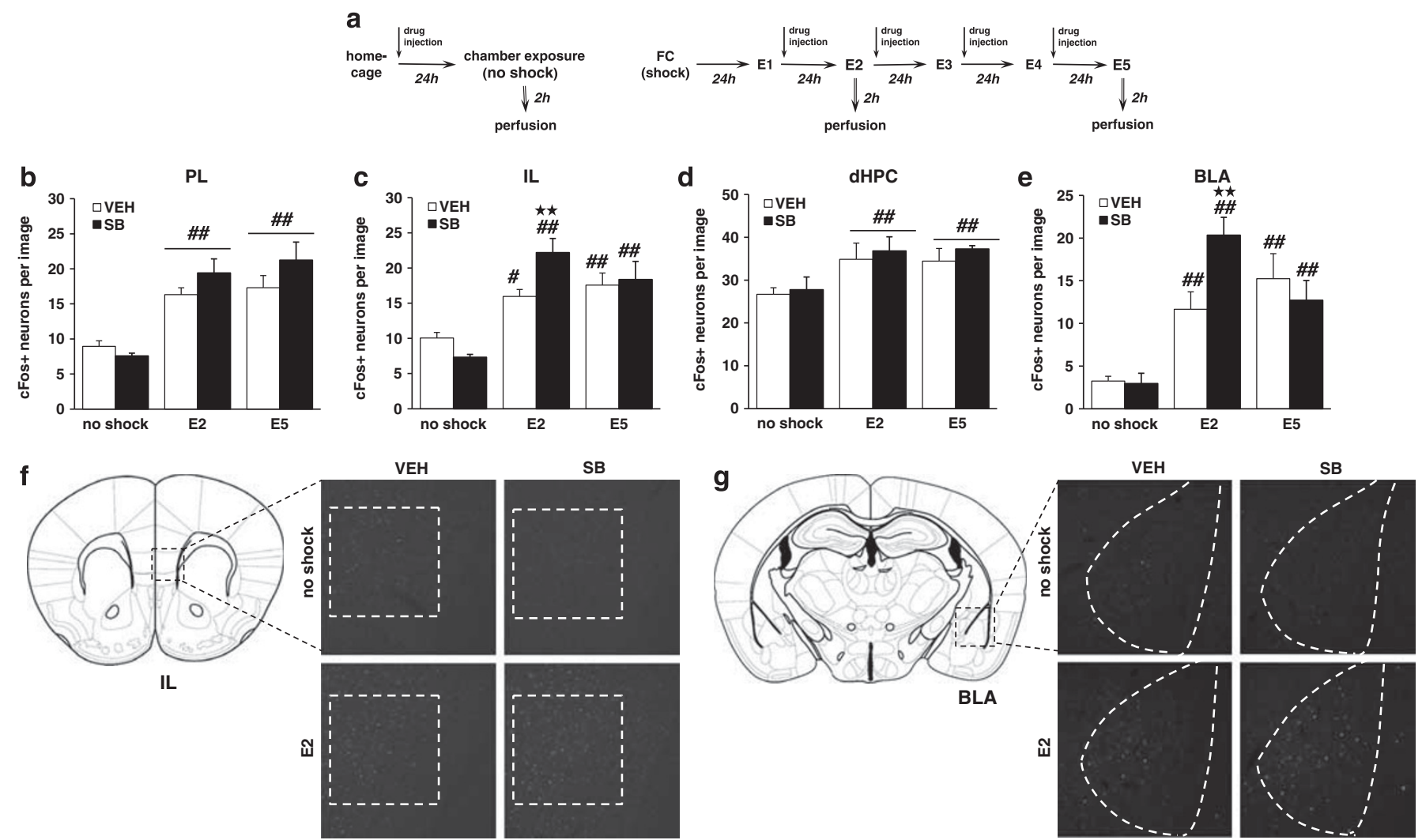

Figure 3 The extinction-facilitating effects of Hcrtr-I blockade are associated with an increased activity of the infralimbic prefrontal cortex and the basolateral amygdala. (a) Schematic representation of the experimental design for (b-g) biochemical experiments. (b-e) Number of neurons expressing cFos in the (b) PL, (c) IL, (d) dHPC, and (e) BLA of mice exposed to the context without receiving footshock, and mice subjected to FC and subsequent extinction until E2 and E5, treated with SB334867 (5 mg/kg, i.p.) or vehicle immediately after each extinction session ( $n=5-8$ mice per group). ( $f$ and $g$ ) Schematic representation of the anatomic location of ( $f$ IL (bregma $+1.54 \mathrm{~mm}$ ) and $(\mathrm{g})$ BLA (bregma— $1.58 \mathrm{~mm}$ ) pictures adapted from Paxinos and Franklin's stereotaxic atlas (Paxinos and Franklin, 200I), and representative images of both regions obtained via fluorescence microscopy after direct labeling with rabbit polyclonal antiserum to cFos. Only no-shock and E2 groups are represented. Quantified areas appear delimited by a discontinuous line. Data are expressed as mean \pm SEM. ${ }^{*} *<0.01$ compared with the vehicle group; ${ }^{*} p<0.05, \# \#<0.01$ compared with the no-shock group. FC, fear conditioning; EI-E5, extinction trials I-5; VEH, vehicle; SB, SB334867; TCS, TCSOX229; PL, prelimbic prefrontal cortex; IL, infralimbic prefrontal cortex; dHPC, dorsal hippocampus; BLA, basolateral amygdala. A full color version of this figure is available at the Neuropsychopharmacology journal online.

in both the amygdala and the hippocampus (Johnson et al, 2012). Interestingly, Hcrtr-1 and Hcrtr-2 antagonists did not modify freezing behavior when administered $4 \mathrm{~h}$ after the FC trial, demonstrating that hypocretin signaling is necessary for the early consolidation of aversive memories. In contrast, recent studies in rats showed a specific role for Hcrtr-1 in the acquisition phase of threat learning (Sears et al, 2013), as revealed by the reduction in freezing levels when SB334867 was injected by i.c.v. route before the auditory FC paradigm. Notably, we did not observe any modification in the consolidation of object-recognition memory due to Hcrtr-1 deletion or pharmacological blockade in contrast to the results of FC, suggesting that hypocretins are mainly involved in the consolidation of highly emotional memories. Consistent with this, the dual hypocretin receptor antagonist DORA-22 did not produce cognitive impairment in the object-recognition test in rats at doses that affected sleep (Uslaner et al, 2013), although contradictory results have been reported regarding the effects of hypocretin antagonists in spatial memory using the Morris water maze (Dietrich and Jenck, 2010; Akbari et al, 2006, 2007). In agreement with a role for hypocretins in threat memories, clinical research has shown abnormal emotional learning in human narcolepsy (Ponz et al, 2010). Moreover, the dual hypocretin antagonist almorexant reduced fear-potentiated startle responses (Steiner et al, 2012), and prepro-hypocretin mRNA levels increased after context-induced FC in rats (Chen et al, 2014). The mechanisms by which Hcrtr-1 regulates the consolidation of fear memories need to be investigated in the future. Hypocretin-containing neurons are sensitive and can also regulate the activity of the corticotrophin-releasing factor (CRF) (Winsky-Sommerer et al, 2004), which is involved in the consolidation of aversive memories (Isogawa et al, 2013). Therefore, the CRF system could be a possible mediator of this effect.

We next evaluated the possible involvement of hypocretins in the extinction of fear memories. For this purpose, Hcrtr-1 and Hcrtr-2 antagonists, and hypocretin-1 and -2 peptides were administered following the formation of aversive memories. In both cued and contextual FC paradigms, we found that Hcrtr-1 blockade with SB334867 strongly facilitated fear extinction consolidation, whereas hypocretin-1 infusion impaired this response. On the contrary, Hcrtr-2 blockade and hypocretin-2 infusion were ineffective, suggesting that hypocretin-1 through Hcrtr-1 activation has a 


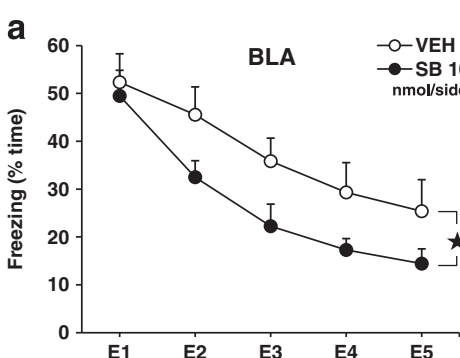

C
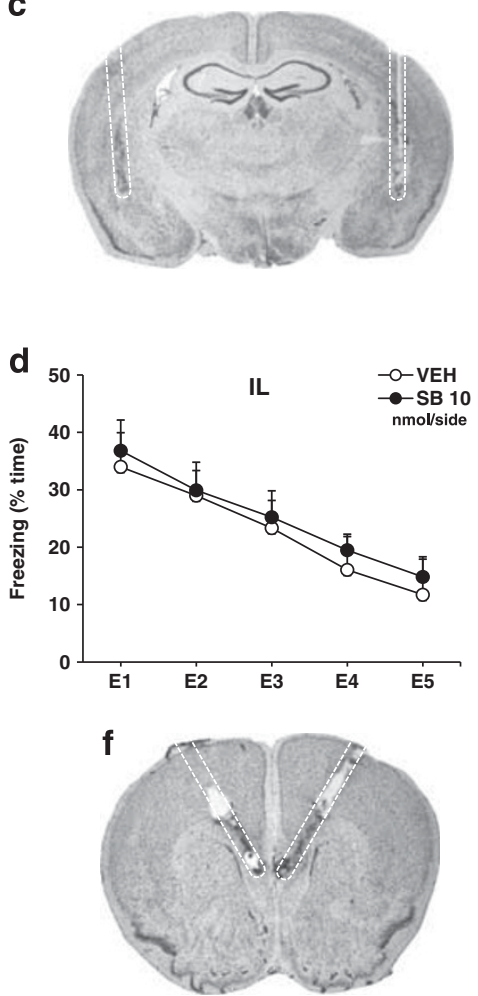
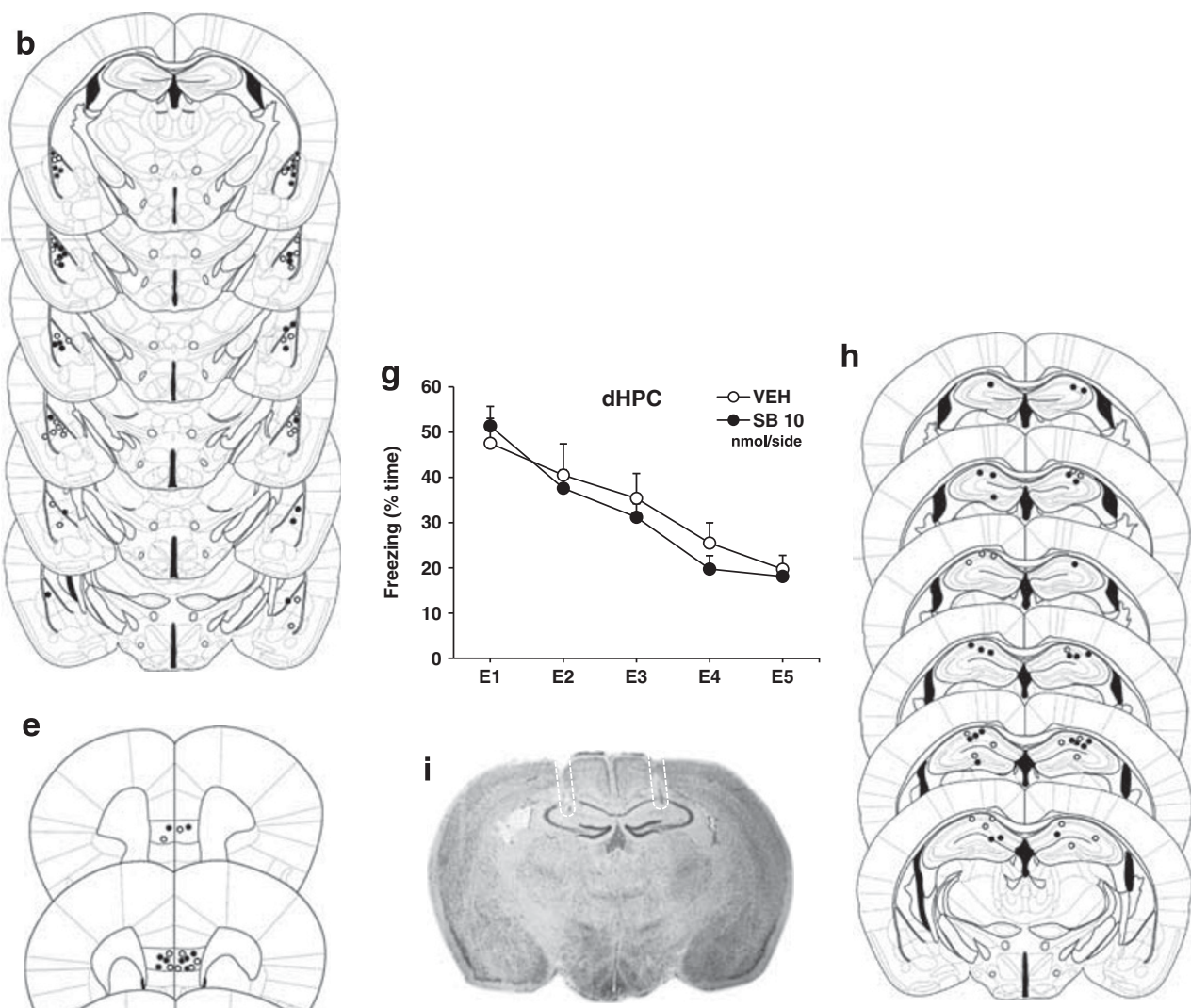

Figure 4 Intra-amygdala infusion of SB334867 facilitates fear extinction. Mice were bilaterally cannulated in the BLA, the IL, and the dHPC and after recovery were subjected to contextual fear conditioning and subsequent extinction procedure. $(\mathrm{a}, \mathrm{d}$ and $\mathrm{g})$ Time course of the freezing levels scored during contextual extinction trials in mice receiving bilateral SB334867 (I 0 nmol/side) microinfusions in the (a) BLA ( $n=\mid 15$ mice per group), the (d) IL ( $n=1 \mathrm{I}-\mathrm{I} 3$ mice per group), or the (g) dHPC ( $n=9-13$ mice per group) immediately after each extinction session. (b,e and $h)$ Location of the vehicle $(O)$ and SB334867 (-) injection sites for each of the mice included in the (b) intra-BLA and the (h) intra-dHPC (between bregma -1.34 and $-1.94 \mathrm{~mm}$ ), and the (d) intra-IL (between bregma +1.78 and +1.42 ). (c, fand i) Representative coronal sections of mouse brains stained with cresyl violet showing the bilateral (c) intra-BLA, (f) intra-IL, and (i) intra-dHPC cannulae placements. Data are expressed as mean \pm SEM. * $p<0.05$ compared with the vehicle group. EI-E5, extinction trials I-5; VEH, vehicle; SB, SB334867; BLA, basolateral amygdala; IL, infralimbic prefrontal cortex; dHPC, dorsal hippocampus.

specific and key role in the extinction of aversive memories. This new physiological function of the hypocretin system was confirmed in the $129 \mathrm{~S} 1 / \mathrm{S} v \mathrm{Im}$ J mouse strain. These mice exhibit a profound impairment of fear extinction (Hefner et al, 2008; Camp et al, 2012) and are considered a valuable model for identifying extinction-facilitating drugs (Whittle et al, 2010; Gunduz-Cinar et al, 2013). We show that Hcrtr-1 blockade rescues deficient extinction of fear memories in $129 \mathrm{~S} 1 / \mathrm{SvImJ}$ mice, suggesting the potential usefulness of this pharmacological target in diseases characterized by inappropriate maintenance of aversive memories. Interestingly, we observed an activation of hypocretin neurons in both the LH and the DMH/PFA during the contextual fear extinction process in C57BL/6J animals, as revealed by double-label immunofluorescence of cFos with hypocretin-1.
Together with the behavioral data, these biochemical results indicate that hypocretin neurons are engaged to preserve fear during the normal physiological process of fear extinction. In addition, chronic hortr-1 blockade could be important to maintain the facilitation of fear memory extinction given that hypocretin neurons remain activated during the whole extinction process.

IL, hippocampus, and amygdala are key structures involved in the neurobiological substrate underlying fear extinction (Herry et al, 2008; Milad and Quirk, 2012). Thus, IL and the hippocampus activate the inhibitory circuits of the amygdala during extinction learning (Herry et al, 2008). Several studies have examined the patterns of the activation of these brain regions during fear extinction by quantifying cFos and Zif268 immediately-early gene (IEG) expression. 
These studies conclude that impaired fear extinction is associated with reduced IEG expression in the corticoamygdala circuit (Holmes and Singewald, 2013; Herry and Mons, 2004; Herry et al, 2010), whereas a complete extinction of conditioned fear is related to increased IEG levels in these brain areas (Herry and Mons, 2004). Several animal models characterized by resistance to extinction such as the 129S1/SvImJ mouse strain (Hefner et al, 2008), mice lacking dynorphin (Bilkei-Gorzo et al, 2012), or a poor-extinction subpopulation of C57BL/6J mice (Herry and Mons, 2004) showed IL hypoactivity, consistent with human studies showing a correlation between patients with PTSD and hypoactivation of the functionally analogous ventromedial prefrontal cortex region (Milad et al, 2009). Likewise, reduced activity of the BLA was found in the same animal models of impaired fear extinction (Hefner et al, 2008; Herry and Mons, 2004; Milad et al, 2009). Two different neuronal populations are activated in the BLA during FC and extinction, and a reduced cFos expression in this area could reflect a failure of the recruitment of extinctionrelated neurons (Herry et al, 2008). Notably, the contextual extinction-facilitating effects of SB334867 was associated with increased cFos expression in both the IL and the BLA, suggesting that the blockage of the hypocretin signaling increases the inhibitory control from the IL over the BLA during this process, and facilitates the activation of the subpopulation of BLA neurons involved in fear extinction. Consistent with this hypothesis, dietary zinc restriction rescued deficient extinction learning and normalized the cortico-amygdala hypoactivity observed in 129S1/SvImJ mice (Whittle et al, 2010). In the present study, SB334867 administration did not modify cFos expression in the PL and dorsal hippocampus. PL has an opposite role to IL and is mainly involved in the formation of aversive memories (Sierra-Mercado et al, 2011).

Hypocretin fibers (Peyron et al, 1998) and Hcrtr-1 (Hervieu et al, 2001; Marcus et al, 2001) show a moderate expression in the BLA, the IL, and the hippocampus. We next evaluated a possible role for Hcrtr-1 in these brain areas in contextual fear extinction. SB334867 infusion in the BLA facilitated extinction of fear memory, whereas this hypocretin antagonist did not produce any effect when administered in either the IL or the dorsal hippocampus. These results suggest a direct involvement of hypocretin activity within the amygdala. Recently, hypocretin signaling in the BLA has been shown to modulate anxious behavior (Arendt et al, 2014), emphasizing the significance of this nucleus in the mediation of hypocretin effects. On the other hand, the lack of effect of SB334867 infusion in the IL suggests that the increased activity of this nucleus associated with the extinction-facilitating effects of Hcrtr-1 blockade is an indirect effect. The paraventricular thalamic nucleus could be one possible candidate to modulate this effect. Indeed, this brain area contains high density of Hcrtr-1 (Marcus et al, 2001), which stimulation modulates fear and anxiety-like behaviors in rodents ( $\mathrm{Li}$ et al, 2010; Hsu et al, 2014), and this nucleus has strong projections to the IL (Vertes and Hoover, 2008). Future experimental work will be necessary to elucidate the brain areas responsible for the disinhibition of the IL induced by SB334867.

In conclusion, our results reveal that hypocretins are selectively involved in the consolidation of highly emotional memories. We show for the first time a key role for the hypocretin system in the BLA in the extinction of fear memories. The possibility of pharmacologically improved extinction of this process is a major uncovered challenge for the treatment of diseases characterized by pathological fear memories, such as PTSD and phobias. These results suggest that Hcrtr-1 blockage could be a promising therapeutical approach for the treatment of these diseases.

\section{FUNDING AND DISCLOSURE}

This work was supported by the Instituto de Salud Carlos III grants, \#PI13/00042 and \#RD12/0028/0023 (RTA-RETICS), by the Spanish Ministry of Science \#SAF2011-29864, the Catalan Government (SGR2009-00731), and by the Catalan Institution for Research and Advanced Studies (ICREA Academia program). África Flores is a recipient of a predoctoral fellowship from the Spanish Ministry of Education. The authors declare no conflict of interest.

\section{ACKNOWLEDGEMENTS}

We thank Marta Linares for invaluable technical assistance.

\section{REFERENCES}

Akbari E, Naghdi N, Motamedi F (2006). Functional inactivation of orexin 1 receptors in CA1 region impairs acquisition, consolidation and retrieval in Morris water maze task. Behav Brain Res 173: 47-52.

Akbari E, Naghdi N, Motamedi F (2007). The selective orexin 1 receptor antagonist SB-334867-A impairs acquisition and consolidation but not retrieval of spatial memory in Morris water maze. Peptides 28: 650-656.

Arendt DH, Hassell J, Li H, Achua JK, Guarnieri DJ, Dileone RJ et al (2014). Anxiolytic function of the orexin 2/hypocretin A receptor in the basolateral amygdala. Psychoneuroendocrinology 40: 17-26.

Bilkei-Gorzo A, Erk S, Schürmann B, Mauer D, Michel K, Boecker $\mathrm{H}$ et al (2012). Dynorphins regulate fear memory: from mice to men. J Neurosci 32: 9335-9343.

Boutrel B, Kenny PJ, Specio SE, Martin-Fardon R, Markou A, Koob GF et al (2005). Role for hypocretin in mediating stressinduced reinstatement of cocaine-seeking behavior. Proc Natl Acad Sci USA 102: 19168-19173.

Camp MC, Macpherson KP, Lederle L, Graybeal C, Gaburro S, Debrouse LM et al (2012). Genetic strain differences in learned fear inhibition associated with variation in neuroendocrine, autonomic, and amygdala dendritic phenotypes. Neuropsychopharmacology 37: 1534-1547.

Chen X, Li S, Kirouac GJ (2014). Blocking of corticotrophin releasing factor receptor-1 during footshock attenuates context fear but not the upregulation of prepro-orexin mRNA in rats. Pharmacol Biochem Behav 120: 1-6.

de Lecea L, Kilduff TS, Peyron C, Gao X, Foye PE, Danielson PE et al (1998). The hypocretins: hypothalamus-specific peptides with neuroexcitatory activity. Proc Natl Acad Sci USA 95: 322-327.

Dietrich H, Jenck F (2010). Intact learning and memory in rats following treatment with the dual orexin receptor antagonist almorexant. Psychopharmacology (Berl) 212: 145-154.

Fanselow MS (1980). Conditioned and unconditional components of post-shock freezing. Pavlov J Biol Sci 15: 177-182.

Flavell CR, Lee JL (2012). Post-training unilateral amygdala lesions selectively impair contextual fear memories. Learn Mem 19: 256-263. 
Flores A, Maldonado R, Berrendero F (2014). The hypocretin/ orexin receptor-1 as a novel target to modulate cannabinoid reward. Biol Psychiatry 75: 499-507.

Gunduz-Cinar O, MacPherson KP, Cinar R, Gamble-George J, Sugden K, Williams B et al (2013). Convergent translational evidence of a role for anandamide in amygdala-mediated fear extinction, threat processing and stress-reactivity. Mol Psychiatry 18: 813-823.

Hefner K, Whittle N, Juhasz J, Norcross M, Karlsson RM, Saksida LM et al (2008). Impaired fear extinction learning and cortico-amygdala circuit abnormalities in a common genetic mouse strain. J Neurosci 28: 8074-8085.

Herry C, Mons N (2004). Resistance to extinction is associated with impaired immediate early gene induction in medial prefrontal cortex and amygdala. Eur J Neurosci 20: 781-790.

Herry C, Ciocchi S, Senn V, Demmou L, Müller C, Lüthi A (2008). Switching on and off fear by distinct neuronal circuits. Nature 454: 600-606.

Herry C, Ferraguti F, Singewald N, Letzkus JJ, Ehrlich I, Lüthi A (2010). Neuronal circuits of fear extinction. Eur J Neurosci 31: 599-612.

Hervieu GJ, Cluderay JE, Harrison DC, Roberts JC, Leslie RA (2001). Gene expression and protein distribution of the orexin-1 receptor in the rat brain and spinal cord. Neuroscience 103: 777-797.

Hollander JA, Lu Q, Cameron MD, Kamenecka TM, Kenny PJ (2008). Insular hypocretin transmission regulates nicotine reward. Proc Natl Acad Sci USA 105: 19480-19485.

Holmes A, Singewald N (2013). Individual differences in recovery from traumatic fear. Trends Neurosci 36: 23-31.

Hsu DT, Kirouac GJ, Zubieta JK, Bhatnagar S (2014). Contributions of the paraventricular thalamic nucleus in the regulation of stress, motivation, and mood. Front Behav Neurosci 8: 73.

Isogawa K, Bush DE, LeDoux JE (2013). Contrasting effects of pretraining, posttraining, and pretesting infusions of corticotropin-releasing factor into the lateral amygdala: attenuation of fear memory formation but facilitation of its expression. Biol Psychiatry 73: 353-359.

Johnson PL, Truitt W, Fitz SD, Minick PE, Dietrich A, Sanghani S et al (2010). A key role for orexin in panic anxiety. Nat Med 16: 111-115.

Johnson PL, Molosh A, Fitz SD, Truitt WA, Shekhar A (2012). Orexin, stress, and anxiety/panic states. Prog Brain Res 198: 133-161.

LeDoux JE (1993). Emotional memory: in search of systems and synapses. Ann N Y Acad Sci 702: 149-157.

Li Y, Li S, Wei C, Wang H, Sui N, Kirouac GJ (2010). Orexins in the paraventricular nucleus of the thalamus mediate anxiety-like responses in rats. Psychopharmacology 212: 251-265.

Li J, Hu Z, de Lecea L (2014). The hypocretins/orexins: integrators of multiple physiological functions. Br J Pharmacol 171: 332-350.

Marcus JN, Aschkenasi CJ, Lee CE, Chemelli RM, Saper CB, Yanagisawa $M$ et al (2001). Differential expression of orexin receptors 1 and 2 in the rat brain. J Comp Neurol 435: 6-25.

Maren S, Phan KL, Liberzon I (2013). The contextual brain: implications for fear conditioning, extinction and psychopathology. Nat Rev Neurosci 14: 417-428.

Milad MR, Pitman RK, Ellis CB, Gold AL, Shin LM, Lasko NB et al (2009). Neurobiological basis of failure to recall extinction memory in posttraumatic stress disorder. Biol Psychiatry 66: $1075-1082$.

Milad MR, Quirk GJ (2012). Fear extinction as a model for translational neuroscience: ten years of progress. Annu Rev Psychol 63: 129-151.

Myers KM, Davis M (2007). Mechanisms of fear extinction. Mol Psychiatry 12: 120-150.
Narita M, Nagumo Y, Hashimoto S, Narita M, Khotib J, Miyatake M et al (2006). Direct involvement of orexinergic systems in the activation of the mesolimbic dopamine pathway and related behaviors induced by morphine. J Neurosci 26: 398-405.

Paxinos G, Franklin KBJ (2001). The Mouse Brain in Stereotaxic Coordinates. Academic Press: San Diego, CA, USA.

Peyron C, Tighe DK, van den Pol AN, de Lecea L, Heller HC, Sutcliffe JG et al (1998). Neurons containing hypocretin (orexin) project to multiple neuronal systems. J Neurosci 18: 9996-10015.

Peyron C, Faraco J, Rogers W, Ripley B, Overeem S, Charnay Y et al (2000). A mutation in a case of early onset narcolepsy and a generalized absence of hypocretin peptides in human narcoleptic brains. Nat Med 6: 991-997.

Plaza-Zabala A, Martín-García E, de Lecea L, Maldonado R, Berrendero F (2010). Hypocretins regulate the anxiogenic-like effects of nicotine and induce reinstatement of nicotine-seeking behavior. J Neurosci 30: 2300-2310.

Plaza-Zabala A, Flores Á, Maldonado R, Berrendero F (2012). Hypocretin/orexin signaling in the hypothalamic paraventricular nucleus is essential for the expression of nicotine withdrawal. Biol Psychiatry 71: 214-223.

Ponz A, Khatami R, Poryazova R, Werth E, Boesiger P, Schwartz S et al (2010). Reduced amygdala activity during aversive conditioning in human narcolepsy. Ann Neurol 67: 394-398.

Sakurai T, Amemiya A, Ishii M, Matsuzaki I, Chemelli RM, Tanaka H et al (1998). Orexins and orexin receptors: a family of hypothalamic neuropeptides and $\mathrm{G}$ protein-coupled receptors that regulate feeding behavior. Cell 92: 573-585.

Scott MM, Marcus JN, Pettersen A, Birnbaum SG, Mochizuki T, Scammell TE et al (2011). Hcrtr1 and 2 signaling differentially regulates depression-like behaviors. Behav Brain Res 222: 289-294.

Sears RM, Fink AE, Wigestrand MB, Farb CR, de Lecea L, LeDoux JE (2013). Orexin/hypocretin system modulates amygdala-dependent threat learning through the locus coeruleus. Proc Natl Acad Sci USA 110: 20260-20265.

Sierra-Mercado D, Padilla-Coreano N, Quirk GJ (2011). Dissociable roles of prelimbic and infralimbic cortices, ventral hippocampus, and basolateral amygdala in the expression and extinction of conditioned fear. Neuropsychopharmacology 36: 529-538.

Soya S, Shoji H, Hasegawa E, Hondo M, Miyakawa T, Yanagisawa M et al (2013). Orexin receptor-1 in the locus coeruleus plays an important role in cue-dependent fear memory consolidation. J Neurosci 33: 14549-14557.

Steiner MA, Lecourt H, Jenck F (2012). The brain orexin system and almorexant in fear-conditioned startle reactions in the rat. Psychopharmacology (Berl) 223: 465-475.

Tronson NC, Corcoran KA, Jovasevic V, Radulovic J (2012). Fear conditioning and extinction: emotional states encoded by distinct signaling pathways. Trends Neurosci 35: 145-155.

Uslaner JM, Tye SJ, Eddins DM, Wang X, Fox SV, Savitz AT et al (2013). Orexin receptor antagonists differ from standard sleep drugs by promoting sleep at doses that do not disrupt cognition. Sci Transl Med 5: 179ra44.

Vertes RP, Hoover WB (2008). Projections of the paraventricular and paratenial nuclei of the dorsal midline thalamus in the rat. J Comp Neurol 508: 212-237.

Whittle N, Hauschild M, Lubec G, Holmes A, Singewald N (2010). Rescue of impaired fear extinction and normalization of corticoamygdala circuit dysfunction in a genetic mouse model by dietary zinc restriction. J Neurosci 30: 13586-13596.

Winsky-Sommerer R, Yamanaka A, Diano S, Borok E, Roberts AJ, Sakurai $\mathrm{T}$ et al (2004). Interaction between the corticotropinreleasing factor system and hypocretins (orexins): a novel circuit mediating stress response. J Neurosci 24: 11439-11448.

Supplementary Information accompanies the paper on the Neuropsychopharmacology website (http://www.nature.com/npp) 